\title{
HF radar observations of ionospheric backscatter during geomagnetically quiet periods
}

\author{
T. A. Kane ${ }^{1}$, R. A. Makarevich ${ }^{2}$, and J. C. Devlin ${ }^{3}$ \\ ${ }^{1}$ Department of Physics, La Trobe University, Bundoora, Victoria, 3086, Australia \\ ${ }^{2}$ Geophysical Institute and Department of Physics, University of Alaska Fairbanks, Fairbanks, AK, 99775-7320, USA \\ ${ }^{3}$ Department of Electronic Engineering, La Trobe University, Bundoora, Victoria, 3086, Australia
}

Correspondence to: R. A. Makarevich (r.makarevich@gi.alaska.edu)

Received: 17 June 2011 - Revised: 22 November 2011 - Accepted: 11 January 2012 - Published: 18 January 2012

\begin{abstract}
The quiet-time coherent backscatter from the Fregion observed by the Tasman International Geospace Environment Radar (TIGER) Bruny Island HF radar is analysed statistically in order to determine typical trends and controlling factors in the ionospheric echo occurrence. A comparison of the F-region peak density values from the IRI2007 model and ionosonde measurements in the vicinity of the radar's footprint shows a very good agreement, particularly at subauroral and auroral latitudes, and model densities within the radar's footprint are used in the following analyses. The occurrence of F-region backscatter is shown to exhibit distinct diurnal, seasonal and solar cycle variations and these are compared with model trends in the F-region peak electron density and Pedersen conductance of the underlying ionosphere. The solar cycle effects in occurrence are demonstrated to be strong and more complex than a simple proportionality on a year-to-year basis. The diurnal and seasonal effects are strongly coupled to each other, with diurnal trends exhibiting a systematic gradual variation from month to month that can be explained when both electron density and conductance trends are considered. During the night, the echo occurrence is suggested to be controlled directly by the density conditions, with a direct proportionality observed between the occurrence and peak electron density. During the day, the echo occurrence appears to be controlled by both conductance and propagation conditions. It is shown that the range of echo occurrence values is smaller for larger conductances and that the electron density determines what value the echo occurrence takes in that range. These results suggest that the irregularity production rates are significantly reduced by the highly conducting E layer during the day while F-region density effects dominate during the night.
\end{abstract}

Keywords. Ionosphere (Auroral ionosphere; Ionospheric irregularities; Plasma waves and instabilities)

\section{Introduction}

Ground-based instrumentation has long been used as a means to study the near-Earth space environment, and it has been said in the past that incoherent scatter radars (ISRs) are the most useful ground-based tool for ionospheric research (Farley, 1996). In part due to high installation and operating costs involved with ISR systems, coherent radars utilizing Bragg scatter from the magnetic-field-aligned irregularities (FAI) in electron density are also frequently used in ionospheric studies. FAIs also represent an integral part of the auroral phenomenology as they are closely related with optical auroral forms, electrojet currents and various plasma instabilities that operate in the auroral region.

The key to coherent radars' operations is their ability to detect backscatter from FAIs. Coherent backscatter occurs when the radar wave is nearly orthogonal to the local magnetic field. At high latitudes, radars operating within the VHF or UHF band are only able to achieve this orthogonality condition at E-region altitudes. Using ground-based, coherent HF radars allows for investigation of both the Eand F-region ionosphere at high latitudes due to larger refraction at HF (Greenwald et al., 1995). The detection of HF backscatter, therefore, depends rather generally on two conditions: (1) the propagation conditions, and (2) the occurrence of ionospheric irregularities.

In the F-region ionosphere, the plasma density irregularities are believed to be driven by the gradient-drift instability (GDI) process. The growth rate of GDI is dependent upon the background electric field, background electron density, and electron density gradients (Keskinen and Ossakow, 1983). Previous studies have also shown that the growth rate of GDI waves in the F-region can be significantly reduced in the presence of a highly conducting E-region, as polarization electric fields associated with the irregularities can be shorted 
through the E-region (Vickrey and Kelley, 1982). Therefore, the second factor above is expected to be controlled by the electric field, electron density and electron density gradients in the F-region, as well as by the electrical conductance of the underlying E-region.

A significant number of past studies have considered theoretical aspects of the GDI wave generation in the F-region (Kelley et al., 1982; Keskinen and Ossakow, 1983; Tsunoda, 1988). The main focus has been on large-scale ( $>1 \mathrm{~km}$ in wavelength) irregularities that are also believed to serve as a seed population on which smaller-scale waves can be generated and be observed directly by coherent radars. Despite a good level of theoretical understanding, it has been noted recently that it is surprising that more detail is not known about small-scale irregularities (Kelley, 2009). This particularly concerns obtaining experimental verification of some of the expected effects including establishing importance of the E-region conductance and scale cascading.

The importance of large-scale irregularities for generation of decameter-scale waves has been noted in some previous studies (Jayachandran et al., 2000; Parkinson et al., 2003), although experimental evidence was difficult to obtain. Similarly, Milan and Lester (1999) and Danskin et al. (2002) have conducted quantitative assessments of the role of the E-region conductance in the power of coherent echoes from the F-region, but results were inconclusive, as often the effect was masked by variations in other parameters such as electric field and electron density. Thus the relative importance of the E-region conductance and other factors has been also difficult to assess.

The electron density in the F-region is important from the point of view of irregularity detection since it controls the amount of refraction experienced by a HF radio wave. Several previous studies employed HF radar data on the echo occurrence and/or power in attempt to quantify this effect using coincident density measurements with ionosondes (Milan et al., 1997) and ISR systems (Milan and Lester, 1999; Danskin et al., 2002) or other considerations of the expected dependencies (Ruohoniemi and Greenwald, 1997; Ballatore et al., 2000; Parkinson et al., 2003; Koustov et al., 2004). In this context, using HF radars of the Super Dual Auroral Radar Network (SuperDARN) offers excellent opportunities to further advance our understanding of factors that control generation and detection of decameter-scale waves in the Fregion. These opportunities are associated with extensive datasets collected over the last solar cycle by most radars in the network.

There have been a number of previous studies that have employed statistical analyses to investigate the F-region backscatter occurrence (e.g. Ruohoniemi and Greenwald, 1997; Milan et al., 1997; Ballatore et al., 2000; Parkinson et al., 2003; Koustov et al., 2004; Wild and Grocott, 2008; Kane and Makarevich, 2010; Kumar et al., 2011). These studies have typically focused on a particular subset of effects, which resulted in a number of other effects remaining coupled. For example, Ruohoniemi and Greenwald (1997) considered the variation in winter backscatter occurrence rates for specific geomagnetic conditions, while Parkinson et al. (2003) considered diurnal variations for several seasons and for all levels of geomagnetic activity as well as for different geomagnetic activity levels but for all seasons combined. The study by Koustov et al. (2004) focused on seasonal dependencies at midnight for all activity levels combined. Also considered were effects of the IMF (Ballatore et al., 2000), magnetospheric substorms (Wild and Grocott, 2008), and geomagnetic storms as defined by storm sudden commencements (Kane and Makarevich, 2010) and temporal filters using Dst time series (Kumar et al., 2011).

The study by Milan et al. (1997) considered arguably the largest subset of factors as they have sorted their radar data according to the universal time, monthly period, and radar slant range, while also providing a density context by considering ionosonde measurements in the E- and F-regions. Milan et al. (1997) also suggested that the amount of backscatter detected by high-latitude HF radars should vary with the solar cycle, as electron density was shown to change considerably from solar maximum to solar minimum, but in their study no HF radar data were available near the solar maximum so no conclusions could be reached on solar cycle effects on the backscatter occurrence.

Although all these approaches have provided valuable insights into both occurrence trends and echo generation mechanisms, a significant scope exists for further efforts to decouple various effects, particularly now that most SuperDARN radars have been collecting good data for many years, enabling investigations into the solar cycle effects. In addition, a focus of some recent studies has been on perturbations in the echo occurrence due to elevated geomagnetic activity, which required information on the quiet-time variation to use as a baseline with which to compare and adjust the disturbed backscatter occurrence (Kane and Makarevich, 2010; Kumar et al., 2011). Thus in order to understand the stormtime variations one must understand in detail the quiet-time backscatter occurrence trends, which has not been investigated before.

In this study, the extensive dataset collected by the SuperDARN TIGER Bruny Island HF radar on the descending leg of solar cycle 23 is employed to analyse quiet-time F-region echo occurrence trends in context of the F-region electron density and E-region conductance. The specific objectives are (1) to examine the solar cycle effects in echo occurrence, (2) to determine and analyse typical patterns in the diurnal and seasonal variations, and (3) to assess relative importance of the electron density and conductance conditions for the F-region echo occurrence. 


\section{Observations}

\subsection{Instrumentation and data post processing}

This study employed data collected with the SuperDARN Bruny Island HF radar which together with the Unwin HF radar in Invercargill, New Zealand forms the dual Tasman International Geospace Environment Radar (TIGER) system (Dyson and Devlin, 2000). These two radars are referred to hereinafter as TIG and UNW, using standard SuperDARN three-letter codes (Chisham et al., 2007). The UNW data were not considered here because this radar commenced operations in late 2004, and its dataset is not long enough for considering solar cycle variations.

The TIG radar is similar, technically and operationally, to all other SuperDARN radars as described in detail by Chisham et al. (2007). The radar consists of 16 antennas which are phased to form an electronically-steerable beam ( $\sim 3^{\circ}$ horizontally, $\sim 30^{\circ}$ vertically) in one of 16 directions. In this study, only data which were collected during either of two common modes (fast or normal) were considered. All 16 beams were scanned sequentially in $60 \mathrm{~s}$ (120 s) for fast (normal) scan providing the spectral power, Doppler velocity, and spectral width measurements in each of 75 range gates separated by $45 \mathrm{~km}$. The first range gate is $180 \mathrm{~km}$.

Since only ionospheric backscatter was of interest to this study, ground- and sea-scatter were removed from the dataset using the standard SuperDARN criteria of low velocity and low spectral width $\left(|| V|-\Delta V|<30 \mathrm{~m} \mathrm{~s}^{-1},|W-\Delta W|<\right.$ $35 \mathrm{~m} \mathrm{~s}^{-1}$, where $\Delta V$ and $\Delta W$ are uncertainties in the velocity and width measurements, respectively. Additionally, all echoes with spectral power less than $3 \mathrm{~dB}$ or spectral width larger than $500 \mathrm{~m} \mathrm{~s}^{-1}$ were also excluded. The same criteria have been employed in numerous previous studies of the F-region backscatter including several recent studies that utilised the TIG data (Kane and Makarevich, 2010; Kumar et al., 2011; Makarevich et al., 2011).

It is typically assumed that HF backscatter received at short ranges $(<765 \mathrm{~km})$ originates from E-region altitudes (e.g. Hanuise et al., 1991; Jayachandran et al., 2000), however some authors have shown that TIG can observe F-region backscatter from as close as $585 \mathrm{~km}$ (Carter and Makarevich, 2009; Makarevich, 2010). To ensure that only backscatter from the F-region ionosphere is considered here, all data from ranges $<765 \mathrm{~km}$ were discarded. Additionally, data from ranges $>3015 \mathrm{~km}$ were also not considered, as these are likely to come from $1 \frac{1}{2}$ - or even $2 \frac{1}{2}$-hop propagation modes. Figure 1 shows the field-of-view (FoV) of TIG, with the dashed lines representing the full radar's FoV and the restricted ranges used in this study $(765-3015 \mathrm{~km})$ shown as solid lines.

One should note here that the standard SuperDARN criteria for separating ionospheric scatter from ground- and sea-scatter are not always most appropriate. Examples of cases when other methods are preferred include backscatter

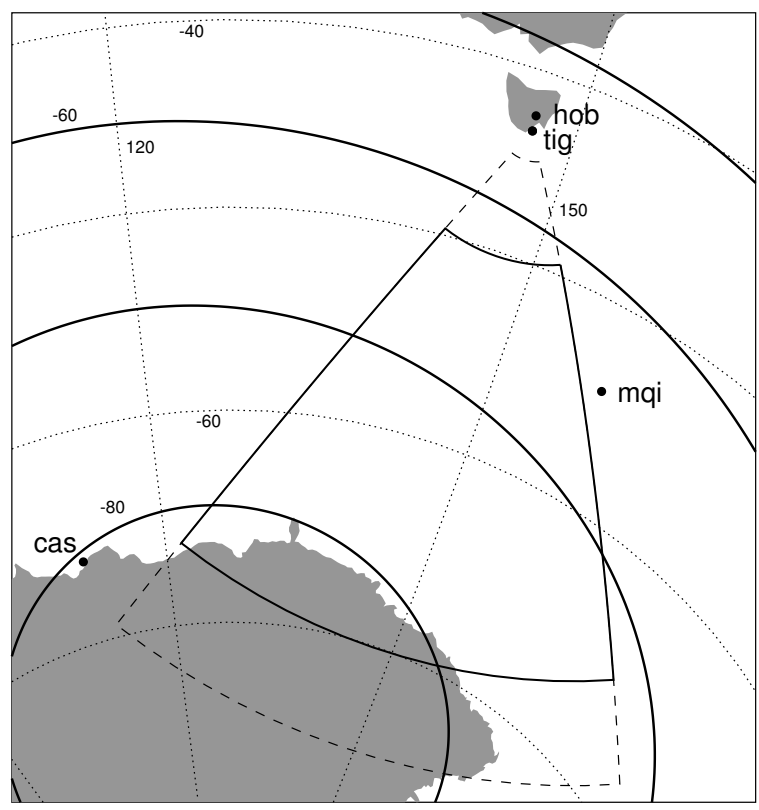

Fig. 1. Field-of-view (FoV) of the TIGER Bruny Island HF radar (TIG). The full FoV is shown by the dashed lines, while the solid lines represent the restricted ranges that were considered in this study. Also shown are the locations of nearby ionosondes: Hobart, Macquarie Island and Casey. Geographic (geomagnetic) coordinates are represented by dotted (bold) lines.

from midlatitude irregularities generated by the temperaturegradient instability (TGI) (Greenwald et al., 2006) and mixed scatter (Ponomarenko et al., 2008). In the most recent study on the issue, Ribeiro et al. (2011) have employed the data from the midlatitude SuperDARN radar at Blackstone, Virginia to develop a new method for identifying TGI-generated scatter detected with midlatitude HF radars (defined in that study as being located at magnetic latitudes lower than $60^{\circ}$ ). Due to its magnetic latitude of $\sim 54.7^{\circ} \mathrm{S}$, the TIG radar is a nominally midlatitude radar. Figure 1 illustrates quite clearly however, that these midlatitude range gates correspond to the shortest ranges which have not been considered in the current study. Regardless, the F-region backscatter (either due to GDI or TGI) is not expected to come from such short ranges.

Only data collected on geomagnetically quiet days were considered in this study. Quiet days were defined here as days on which the magnitude of the geomagnetic Dst index was below $30 \mathrm{nT}$ ( $|\mathrm{Dst}|<30 \mathrm{nT}$ ) for the entire day. This definition is consistent with the general view of geomagnetically quiet periods as intervals with no storm activity, where the minimum daily Dst value of $-30 \mathrm{nT}$ is used to define weakest storms (Gonzalez et al., 1994). It is also the same definition as that employed by Kane and Makarevich (2010).

To investigate controlling factors of the quiet-time backscatter occurrence, measurements of F-region critical frequency $(f o \mathrm{~F} 2)$ and hence F-region peak electron density $(N m \mathrm{~F} 2)$ are desirable. The locations of the 3 nearest 


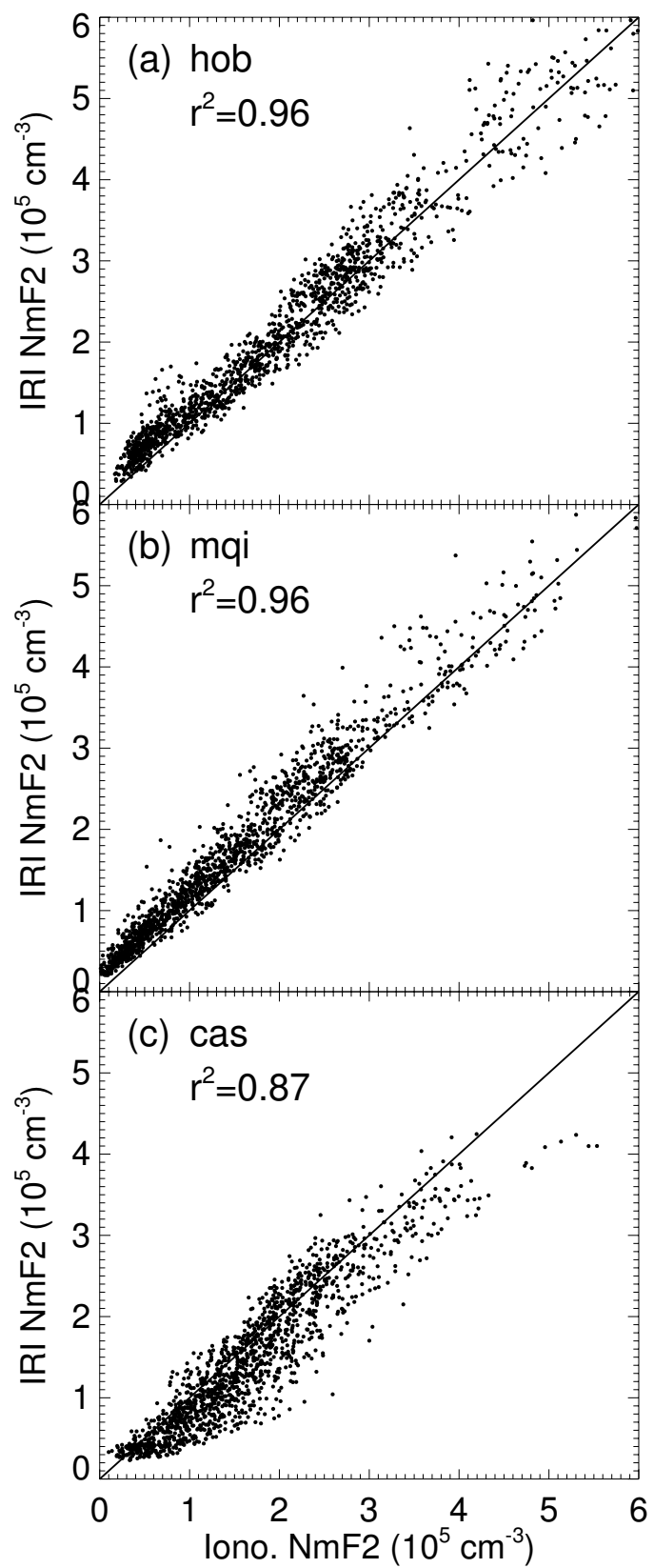

Fig. 2. Comparison of ionosonde measurements of $N m \mathrm{~F} 2$ with values taken from the IRI model with the ionosonde locations used as an input. The ionosonde code is shown in the top-left of each panel. The linear correlation co-efficient $r^{2}$ is shown under the ionosonde code.

ionosondes to the TIG FoV are also shown in Fig. 1. It shows that the ionosonde measurements of $f o \mathrm{~F} 2$, and hence $N m \mathrm{~F} 2$, are not exactly coincident with the TIG observations. In order to obtain values for $N m \mathrm{~F} 2$ within the $\mathrm{FoV}$, the latest version of the International Reference Ionosphere (IRI2007) model (Bilitza and Reinisch, 2008) was used to produce $N m$ F2 for each range-beam cell within the TIG FoV. The IRI-2007 model was also used to calculate $N m$ F2 at the ionosonde locations which were then compared with the ionosonde data in order to validate our use of the model. The results of this validation analysis are presented in Sect. 2.2, while the obtained model values are used in the analysis presented in Sect. 2.4.

In addition to the electron density in the F-region, a potentially important controlling factor to consider is an ionospheric electrical conductance. The Pedersen conductance estimates used in this study were obtained from the model by Robinson and Vondrak (1984). That study employed the electron density profiles measured by an ISR system and modelled other parameters to determine electrical conductivities in the vicinity of the $\mathrm{E}$ layer. These conductivities were integrated over heights of $90-200 \mathrm{~km}$ and the resulting conductances were compared to the solar zenith angle and $10.7-\mathrm{cm}$ radio flux in order to derive a model relationship of the form

$\Sigma_{P}=0.88 \sqrt{S_{a} \cos \chi}$

where $\Sigma_{P}$ is the Pedersen conductance, $S_{a}$ is the $10.7-\mathrm{cm}$ solar radio flux, and $\chi$ is the solar zenith angle. A very similar relationship was derived for the Hall conductance, where a co-efficient of 1.5 was obtained (instead of 0.88 ). Therefore, the Hall and Pedersen conductances are simply proportional to each other in this model, and in the current study, the model Pedersen conductance was computed for all rangebeam cells within the restricted FoV, and for each hourly time interval with given values of $S_{a}$ and $\chi$. The resulting set of values was averaged over the entire FoV and the results presented in Sect. 2.4.

\subsection{Comparison of ionosonde measurements with IRI-2007}

In the absence of coincident electron density measurements, the IRI-2007 model was implemented to determine the values for $N m \mathrm{~F} 2$ within the TIG FoV. In order to justify the use of the model for our purposes, a comparison is made with the data from the ionosondes, Fig. 1. The ionosonde $f o \mathrm{~F} 2$ data were available with a resolution of $1 \mathrm{~h}$ and $N m \mathrm{~F} 2$ was determined using the relationship. $N_{\mathrm{e}}=1.24 \times 10^{4} f^{2}$, where $N_{\mathrm{e}}$ is in units of $\mathrm{cm}^{-3}$ and $f$ is in $\mathrm{MHz}$.

The IRI-2007 model was run at the ionosonde locations on all days in 2002-2006 for which ionosonde data were available, using the URSI- 88 co-efficients for $f o F 2$. Figure 2 shows the comparisons between the $N m \mathrm{~F} 2$ values obtained from IRI-2007 and from the 3 nearby ionosondes. The linear Pearson correlation co-efficient is shown in the top-left of each panel, under the ionosonde code. In all 3 cases, the modelled and measured values were well correlated, with $r^{2}$ between 0.87-0.96. An excellent correlation of 0.96 was obtained at subauroral (HOB) and auroral (MQI) stations, while at a high-latitude station (CAS) the correlation was lower.

The excellent agreement between quiet-time model values and ionosonde measurements presented in Fig. 2a and 
b shows that the IRI-2007 model values accurately represent the values of $f_{o} \mathrm{~F} 2$, and hence $N m \mathrm{~F} 2$, at the subauroral and auroral latitudes. It is from these latitudes that TIG receives most echoes according to Parkinson et al. (2003) and therefore where the propagation conditions are most likely to affect backscatter detection rates. As such, the IRI-2007 model is used for the remainder of the present study, as the model can be run at locations within the TIG FoV, allowing backscatter occurrence data to be compared with coincident information about the electron density.

\subsection{Quiet-day-curve algorithm}

The quiet-time backscatter data used in this study are analogous to the quiet-day curves (QDCs) employed by Kane and Makarevich (2010). In that previous study, QDCs were used as a baseline trend for adjusting the storm-time backscatter occurrence, whereas the present study focuses specifically on these geomagnetically quiet periods. As such, the algorithm used to process the TIG data in this study, and indeed for processing the electron density and conductance model values, is the same as the QDC computation method used in that previous study. The parameters considered here are: (1) F-region backscatter occurrence, (2) mean slant range of F-region echoes, (3) F-region peak electron density $N m \mathrm{~F} 2$, and (4) Pedersen conductance $\Sigma_{P}$.

For each quiet day within the dataset, a daily trend at 1$h$ resolution was produced for each parameter. For the TIG data (which has a higher temporal resolution) the value for each hour was taken to be the total number of echoes detected within the entire F-region FoV (ranges 765-3015 km) divided by the number of range-beam cells $(16$ beams $\times 50$ range gates $=800$ range-beam cells) within the F-region FoV. The number of echoes detected by TIG was normalised to a 1-min mode by doubling the number of echoes detected for 2-min modes. The model $N m \mathrm{~F} 2$ and $\Sigma_{P}$ values were calculated for the start time of each hour, and these values were used as representative values for the entire hourly period.

\subsection{Quiet-time backscatter occurrence in the F-region}

The TIG data were analysed using the algorithm described above to examine solar cycle, seasonal and diurnal effects in echo occurrence. The results of this analysis are presented in the first row of Fig. 3. Each panel of the figure represents one year, as indicated at the top of the column. The horizontal axis of the figure represents the month, and the vertical axis represents the hour in local time (LT). Each cell within a panel, therefore, represents the typical amount of backscatter one might expect to observe during quiet times for a given hour of local time during a given month. The number of echoes per range-beam cell of the FoV is indicated by the color of the cell, and the scale is indicated to the right of the figure. A related parameter of the mean slant range of ionospheric echoes over the considered viewing area is shown in the second row of Fig. 3. Each cell within a second-row panel represents a typical backscatter location for a given LT and a given season. The solid white lines in each panel in the first 3 rows of Fig. 3 represent a terminator line at an F-region altitude of $300 \mathrm{~km}$.

Several features are immediately obvious from Fig. 3 . First, following the vertical axis from 00:00 to 24:00 LT, the diurnal variations are quite clear. Further, the nature of the diurnal variation itself varies with month (season). It can be seen that during the southern summer, there are large amounts of backscatter detected in the late evening to early morning sectors with a distinct depletion of backscatter occurrence during the noon sector. During the winter, on the other hand, TIG observed fewer echoes during the midnight and early morning sectors. The timing of the maximum in backscatter occurrence moves to earlier local times during the winter months, as compared with summer.

Importantly, this type of presentation clearly shows a gradual progression of the echo occurrence maximum from midnight in summer to midday in winter with associated splitting into two peaks in between these seasons. The two areas of high occurrence (green-to-red cells) form a "butterfly" or "hour glass" shape which is slightly asymmetric with respect to the middle vertical line at $x=6$, with the right part of the pattern being somewhat weaker in 2002-2004.

The coupled nature of the diurnal and seasonal variations is related with the solar zenith angle conditions as indicated by the terminator line generally following the "butterfly" shape of the enhanced backscatter area in Fig. 3. In 20022003, the backscatter is strongly enhanced on both sides of the terminator line in the pre-midnight sector including summer months, while in the post-midnight sector it was mostly observed after sunrise, with before-sunrise enhancements limited to winter months. During later years 20052006, the backscatter mostly falls between the two terminator lines, i.e. it was mostly observed during the daytime.

During the period under consideration, the solar activity decreased from near solar maximum in 2002 to near solar minimum in 2006. Overall, the solar cycle dependence appears to be a simple decrease in occurrence throughout the study interval. This is reflected in both weakening of the high-occurrence "butterfly" areas and further depletion of low-occurrence triangular areas at the top and bottom of each panel. Interestingly, the other low-occurrence triangular "bite-out" areas on the left and right of each panel do not seem to change much from 2002 to 2006.

The mean slant range of ionospheric echoes (Fig. 3, second row) also exhibits distinct diurnal and seasonal trends so that the contours of equal range follow the "butterfly" shape or terminator lines. The backscatter was detected farther from (closer to) the radar during daytime (nighttime) hours, pointing towards the importance of propagation conditions for backscatter detection and location as well as once again supporting the notion of significantly different character of the daytime and nighttime backscatter. The implications of 


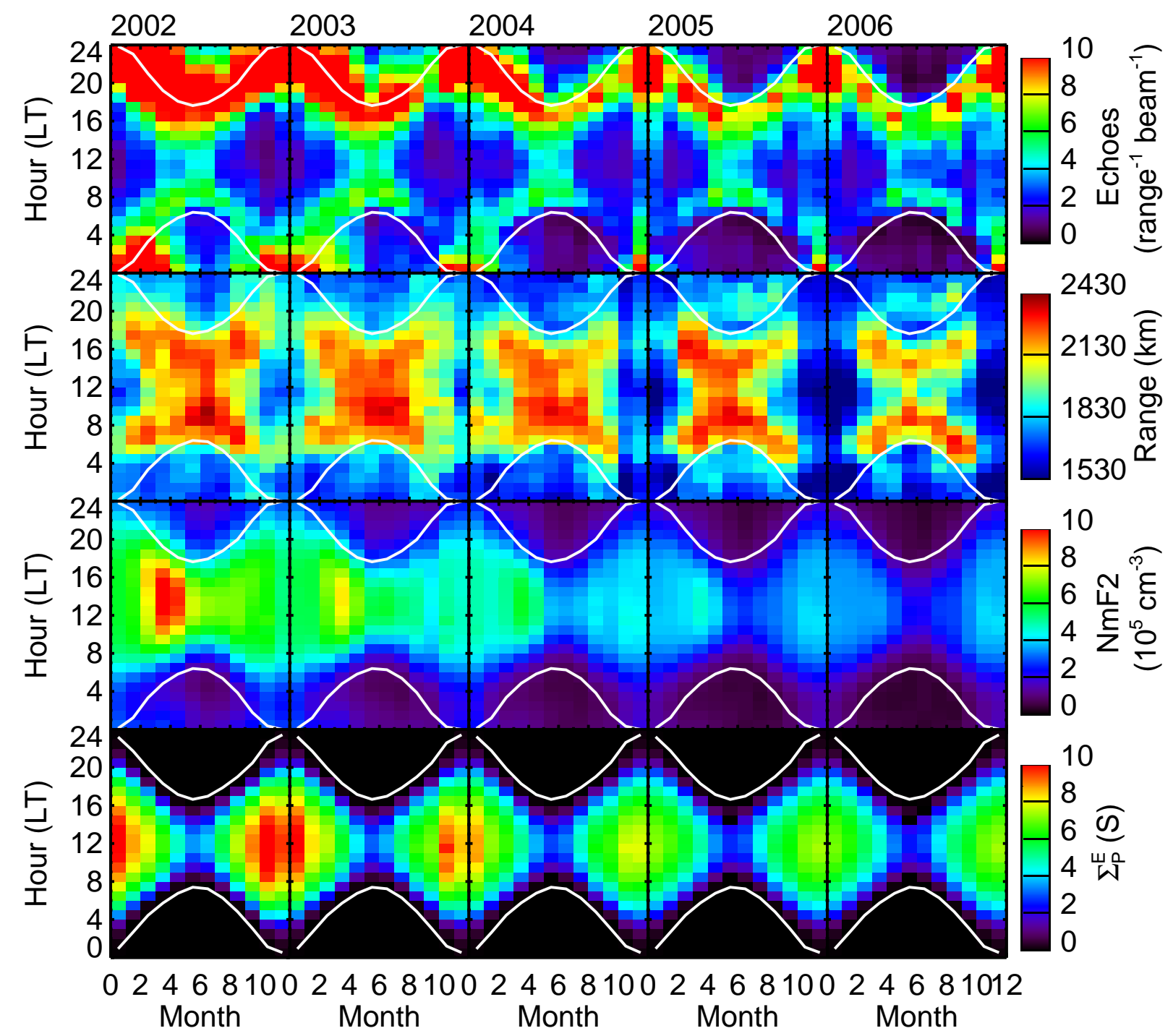

Fig. 3. Quiet-time statistics for the parameters considered in this study. Each column of panels represents one year of data, as indicated at the top of the column. Each row of panels represents one of the parameters. These parameters are from top to bottom: the radar echo occurrence, average slant range of the ionospheric echoes, model $N m \mathrm{~F} 2$ and model conductance. The horizontal (vertical) axis of each panel shows the month (local time) of the data shown in each cell. The color code for each parameter is indicated to the right of the figure. The white lines in the 3 top rows ( 1 bottom row) represent a terminator line at an F-region (E-region) altitude of $300 \mathrm{~km}(110 \mathrm{~km})$.

the observed variations in typical backscatter location are further discussed in Sect. 3.5.

In order to facilitate an investigation into the factors that control the changes in backscatter occurrence, Fig. 3 also presents the variations in the modelled values of the F-region electron density and the Pedersen conductance over the same period. Both of these parameters are analysed in the same manner as the radar backscatter, using the algorithm described above. The third row of the figure shows the $N m \mathrm{~F} 2$ parameter averaged over the TIG FoV and the bottom row shows the average Pedersen conductance. The layout of these plots is the same as the occurrence panels, with only the plotted parameter and color scale being different.
As with the echo occurrence, the electron density values exhibit distinct diurnal, seasonal and solar cycle variations. As expected, the electron density is enhanced under sunlit conditions, between the terminator lines. It is largest in the noon sector, when the solar zenith angle is low and the rate of photo-ionisation is high. The seasonal variation of $N \mathrm{mF} 2$ shows that the increase in density with LT begins later in the morning during the winter than during the summer, due to the solar zenith angle conditions. The density decreases with the solar cycle and a nighttime bite-out is observed to become more extended in season and LT as solar cycle weakens, similar to that shown in occurrence. The bite-out areas (at the top and the bottom) in occurrence and density are 
actually very similar, including the fact that they are quite restricted in 2002 (to summer and LT around $\sim 4 \mathrm{~h}$ at the bottom) but become more extended together (in both rows) as solar cycle progresses. One should also note that the lowoccurrence areas on the left and right of all panels in the top row are very different in that they actually correspond to the enhanced density areas in the middle row.

The average Pedersen conductance appears to behave in a similar manner to the F-region electron density, with a peak near noon and a strong seasonal dependence. Since the conductance model (Eq. 1) takes as an input the solar zenith angle, the conductance decreases sharply at dusk and become zero during the night, so that non-zero conductance area in the bottom row is located strictly between the terminator lines at $110 \mathrm{~km}$. The solar flux input in the conductance model leads to the strong solar cycle dependence of conductance. From the conductance consideration, it appears that the low-occurrence areas on the left and the right correspond better to the enhanced conductance (rather than to the F-region density which is also enhanced). Hence this occurrence depletion is observed during the daytime conditions.

\section{Discussion}

\subsection{IRI model validity}

The $N m$ F2 values determined from the IRI-2007 model were shown to be in quite good agreement with those measured by ionosondes in the vicinity of the TIG FoV, as presented in Fig. 2. A particularly good agreement was observed at subauroral and auroral latitudes. The IRI model describes ionospheric densities and temperatures in the non-auroral ionosphere (Bilitza and Reinisch, 2008) and hence an excellent agreement at subauroral latitudes during quiet conditions is expected. The fact that the same amount of correlation was also observed at a significantly more poleward location of MQI implies that the IRI model worked reasonably well in this large region spanning nominally subauroral and auroral latitudes during the considered quiet periods.

The IRI model predictions are generally less accurate in the regions where ionosonde coverage is more sparse (Bilitza and Reinisch, 2008). For this reason, the IRI's somewhat poorer performance at a high-latitude station of CAS is not surprising, although a relatively high correlation value of 0.87 means that the deterioration was not significant enough to invalidate our approach of considering the TIG FoV up to ranges of $3015 \mathrm{~km}$. At the same time, not considering farther ranges $(3015-3600 \mathrm{~km})$ is probably a good choice even if only for the reason of a slightly poorer IRI performance.

The conductance model used here (Robinson and Vondrak, 1984) was based specifically on geomagnetically quiet days, i.e. under conditions when solar illumination was the dominant source of ionisation. In fitting the model to their data, Robinson and Vondrak (1984) discarded data for which the
$N m$ E parameter was large due to particle precipitation. In the present study, only quiet days were considered so it is safe to assume that the Pedersen conductance is controlled by photoionisation rather than particle precipitation. A comparison of the IRI-derived $N m \mathrm{E}$ (using the algorithm described in Sect. 2.3) with the model conductance values shown in Fig. 3 yielded a correlation value of $r^{2}=0.99$ (data not shown). This validates this assumption, and hence the use of this model.

Strictly speaking, the Robinson and Vondrak's (1984) model is not valid for zenith angles greater than $85^{\circ}$. This consideration was not taken into account in this study, and zenith angles up to $90^{\circ}$ were included. Zenith angles larger than $90^{\circ}$ resulted in a conductance of zero. Since averages were taken over every range-beam cell within the TIG FoV, this has resulted in very small, non-zero values for conductance being shown for summer midnight. A related issue is that, physically, the conductance should not decrease to zero during the night. Some previous studies involving this model have taken this into account by adding a constant offset to the model to give a non-zero nighttime conductance (Benkevitch et al., 2002). Since this would have only a minor effect on the conductance values and since the present study was mostly concerned with qualitative behaviour of occurrence, density, and conductance, this effect was not considered in the present study.

The 10.7-cm solar flux (F10.7) was used as a proxy for the EUV flux, as the F10.7 is measured from the ground and the relationship between F10.7 and the EUV flux is typically assumed to be linear. Some previous studies have shown that for large solar activity the assumption of linearity between these fluxes does not hold as the EUV flux becomes "saturated" beyond some key threshold. The maximum threshold for which F10.7 may be assumed to vary linearly with EUV flux varies, but is usually cited at around 200 Solar Flux Units (SFU) (Balan et al., 1993). In 2002, some days had F10.7 up to, and slightly in excess of, 200 SFU which could potentially lead to a slight over-estimation of the conductance shown in Fig. 3 for 2002. An excellent agreement between the E-region peak density measured by ionosondes and model conductance (data not shown) suggests that this was not significant. This saturation is taken into account in the IRI model, and so the F-region electron densities used here are valid.

\subsection{Solar cycle effects}

The solar cycle effect was observed in all parameters considered in Fig. 3 as a general decrease in the numbers from 2002 to 2006. The effect observed in the model F-region density and Pedersen conductance is a result of the general decrease of solar EUV flux with solar activity from the maximum to minimum years studied here. The conductance decreased from year to year, but the LT at which it decreases to near zero is not affected by the solar cycle. Since the zenith 


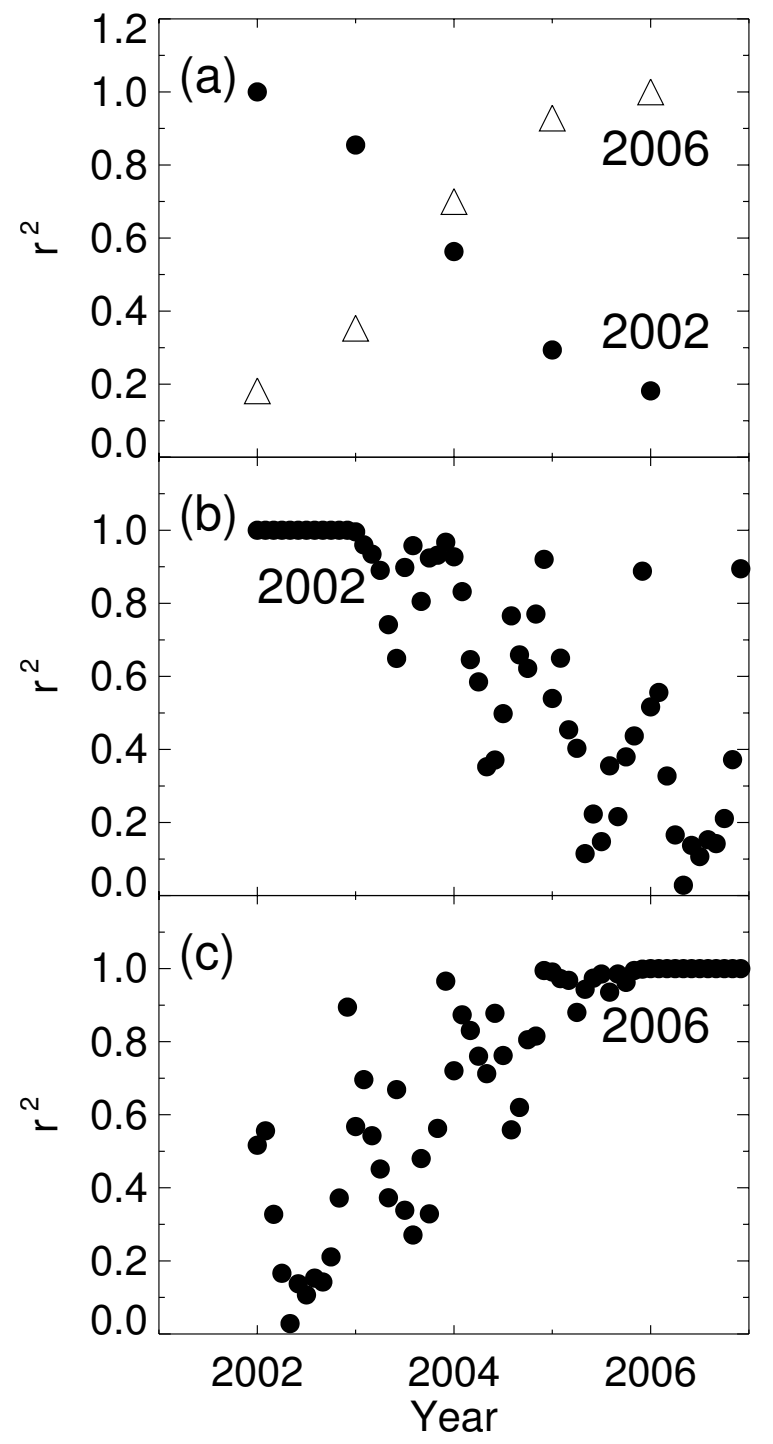

Fig. 4. Correlation analysis of the solar cycle dependence of the echo occurrence. Circles (triangles) in panel (a) show correlation co-efficients between the occurrence values in 2002 (2006) and all considered years from Fig. 3. Panels (b) and (c) are the same as, respectively, circles and triangles in panel (a) except that occurrences for each month were cross-correlated here between different years.

angle, which obviously does not depend on solar cycle, is an input in the model one should not expect to see a solar cycle dependence in the timing of minima and maxima. The agreement between the E-region peak density and conductance (data not shown) would suggest that this is a physical effect, and not simply a model limitation.

An important question with regard to this effect is whether a decrease in occurrence from year to year can be simply described as a "proportionality" effect, that is whether the pattern in 2002 is essentially the same as in 2006, but with all values multiplied by a certain factor. One feature that was mentioned before, that the low-occurrence areas on the left and right sides of each pattern did not change much qualitatively, unlike their bottom and top counterparts as well as the high-occurrence areas, did indeed suggest as much.

One quantitative way to further assess this is by conducting a cross-correlation analysis between occurrence patterns in different years. Figure 4 shows the results of this analysis. Panel (a) shows the linear Pearson correlation co-efficients between occurrence values in 2002 with those in other years by circles. Triangles show the same correlations but between the year 2006 and other years. If the patterns were simply proportional to each other, then both trends would be approximately flat with correlations near 1 . This is clearly not observed here. Instead, both trends are clearly monotonic, which gives a strong indication that the occurrence patterns cannot be described using a proportionality rule. The fact that one curve is increasing in Fig. 4, while the other one is decreasing with year implies in fact that the solar cycle effect is strong but nonlinear, with distinct trend from one year to another. That is the pattern changes in a systematic way when the first-order proportionality effect is removed (by considering correlations or, for example, by normalizing all patterns to the maximum over the entire year).

Figure $4 \mathrm{~b}$ and $\mathrm{c}$ shows a similar analysis only with correlations for each month between all years. For example, the first (last) circle from the left in panel (b) shows a correlation co-efficient between occurrence values in January (December) 2002 and all years for a month of January (December). To be consistent with panel (a), correlations of 1.0 between the same datasets are also shown. Here one can also notice a seasonal variation associated with sinusoidal variations in the correlation co-efficients with time. This analysis also shows that the correlations decrease or increase in a systematic way for the same month, which again implies a strong nonlinear nature of solar cycle effects.

The solar cycle effects in the radar echo occurrence were not considered in the previous studies, due to limited data availability at the time. The new result of the current study is therefore that these effects are strong and moreover strongly nonlinear.

\subsection{Seasonal and diurnal effects: a coupled picture}

Apart from the solar cycle effects, the two other effects present in Fig. 3 were the seasonal and diurnal variations. Again, these effects were clearly present in all parameters considered.

The variations of both ionospheric electron density and conductance shown in Fig. 3 (third and fourth rows) can be readily explained by solar illumination (in the case of conductance it is trivial as zenith angle is a model input). During the day, both parameters increase maximising around noon and decrease during the night. The seasonal effect is also clear, with both parameters remaining high for larger lengths of time during the summer than in the winter due to the high latitudes being sunlit for longer periods of time during the 
summer. The rate at which the parameters decrease in the afternoon and evening differs for the density and conductance, however. The Pedersen conductivity peak is much closer in height to the E-region and the rate of recombination is higher than in the F-region. The E-region electron density will therefore decrease rapidly once production stops, resulting in a decrease in conductance beginning at noon. The slow recombination in the F-region on the other hand, allows the electron density to remain high into the afternoon and evening sectors.

Perhaps the most relevant previous study to the present investigation is the occurrence component of a study by Parkinson et al. (2003). The seasonal variation of occurrence shown in Fig. 3 (top row) is in good qualitative agreement with the seasonal trends presented there. Thus Parkinson et al. (2003) reported that the main echo band was more extended in LT during winter than during summer, which is what is observed in our case as well. However, our observations also showed that there is a gap in echo occurrence near 00:00 MLT in winter, which was not reported by Parkinson et al. (2003). In fact, their Fig. 4 shows that instead of a gap the occurrence simply decreases after 00:00 MLT with LT, without picking up again near 08:00 MLT as it does in our case. One reason why this may have happened is that Parkinson et al. (2003) considered seasonal variation for all geomagnetic conditions together, while the present study is only concerned with the quiet conditions.

The other important difference between these two studies is in the employed approach. While Parkinson et al. (2003) effectively takes seasonal "snapshots" by considering 4 seasons separately, our study considers a gradual transition between them simultaneously with similarly-gradual diurnal variation. In this sense, the present approach is different from those in most other previous investigations. This approach allowed to identify new features such as two cases of diurnal variation: with single, pre-midnight peak in summer and with two peaks pre- and post-noon in winter and a gradual transition between them with season associated with movement of the peaks in LT. Moreover, the explanation of this "coupled" behaviour may lie in the density and conductance control of the occurrence patterns as described below.

\subsection{Seasonal and diurnal effects: density and conduc- tance control}

A visual inspection of Fig. 3 suggests that the daytime minimum in F-region backscatter occurrence is correlated with the conductance maximum. However, the F-region electron density is also at maximum during the day. In order to investigate the relative control of occurrence by conductance and electron density, we now consider day- and nighttime backscatter separately.

For the purposes of the following discussion we define the night time as periods when the Pedersen conductance is zero, and the day time as all other times. As mentioned in Sect. 3.1,

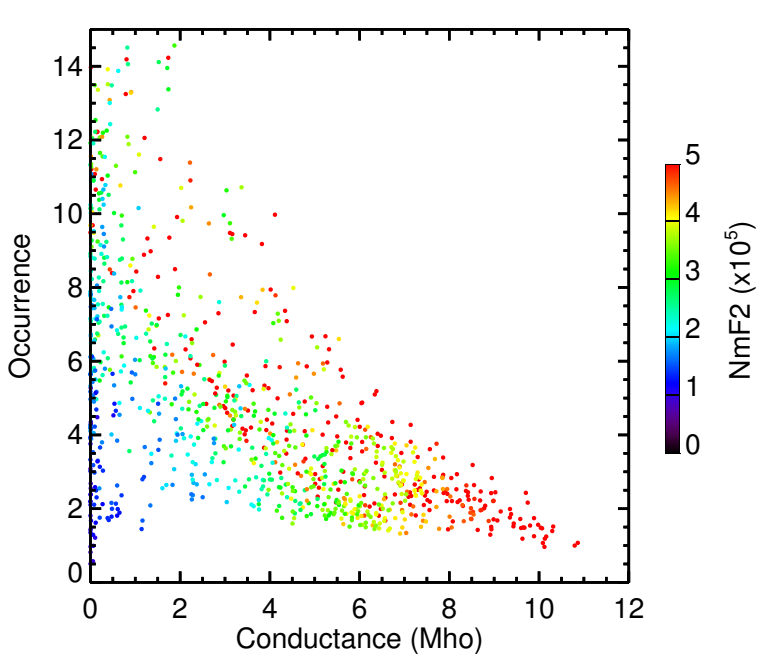

Fig. 5. Daytime radar echo occurrence versus model Pedersen conductance. Each point is color-coded according to the corresponding value of $N m \mathrm{~F} 2$, with the scale shown on the right.

the summer midnight has very small, non-zero values due to the higher latitudes within the TIG FoV being included in the average values. These values will therefore be included in the daytime dataset based on this selection criterion.

Figure 5 shows the echo occurrence versus conductance for the same dataset as that used in Fig. 3 (nighttime observations excluded). Each point corresponds to one month-hour cell in Fig. 3; it is color-coded in the corresponding electron density.

The points in the left part of the figure with near-zero conductance are mostly the nighttime data points as discussed above. The grouping of these points near the vertical axis shows that their contamination of the daytime dataset is not significant. The figure shows that for higher conductance values, the amount of backscatter detected decreases significantly. The absence of data points in the top-right part of the figure is, in itself, evidence that high conductance suppresses F-region irregularities, since high occurrence rates simply were not observed by the radar during the periods of high conductance.

It is important to note that for low conductance the amount of backscatter detected can be large or small, so that the conductance of the underlying ionosphere appears to strongly control the amount of F-region backscatter (irregularities) only when it is high. The theoretical predictions of Vickrey and Kelley (1982) show that for high Pedersen conductance one would expect a suppression of F-region irregularity generation for large-scale irregularities. The results shown in Figs. 3 and 5 suggest that this also holds true for decameterscale irregularities, and that the mechanisms described by Vickrey and Kelley (1982) are likely to be responsible for the low daytime occurrence observed here. Of course it is impossible to conclude from our data whether the effect is direct, 


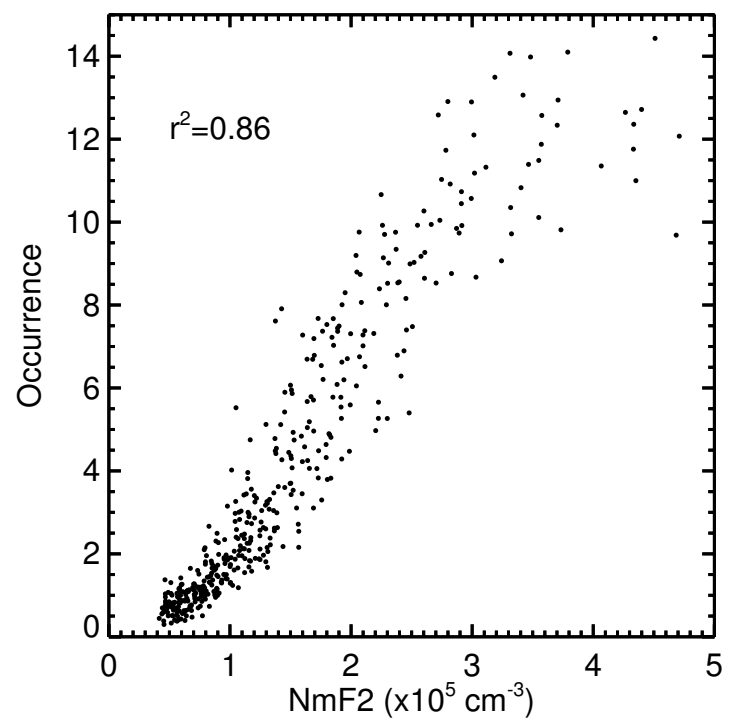

Fig. 6. Nighttime echo occurrence versus model F-region electron density $N m \mathrm{~F} 2$. The linear $r^{2}$ correlation co-efficient is shown in the top-left of the figure.

i.e. when the electric fields associated with decameter-scale perturbation are shorted by the highly conducting E-region, or indirect, i.e. when large-scale perturbation electric fields are shorted so that small-scale irregularities have no largescale seed population to thrive upon.

Even though the suppression effect is maximised at very high conductances in excess of 8 Mhos, the data presented in Fig. 5 are consistent with the idea that the conductance control exists at all conductance values, as the scatter pattern has a well-defined upper envelope, which drops with conductance. The lower envelope is also evident in Fig. 5 only with a much lower rate of decrease.

Figure 5 also shows a well-defined pattern in the color coding, with higher density points generally located higher for the same conductance value. This indicates that in addition to the conductance control, the F-region density also has an effect on the daytime occurrence. This effect is likely to be due to more favourable propagation conditions for a certain density (Danskin et al., 2002). Sorting the data simultaneously in both conductance and density appears to emphasise both effects during the daytime conditions. The new result obtained using this approach is that a certain range of densities would produce a different range of occurrence values depending on the conductance.

During the night, when modelled (physical) conductance is zero (low), the conductance should not be expected to suppress the generation of F-region irregularities. Figure 6 shows the echo occurrence versus the electron density in order to investigate the nighttime bite-out present in Fig. 3. The data points in this figure correspond to month-hour cells in Fig. 3 in which the corresponding conductance value was zero.
Figure 6 also shows the linear correlation co-efficient in the top-left of the figure. This correlation was quite high, $r^{2}=0.86$, which indicates that, in the absence of conductance effects, the amount of backscatter detected appears to be directly proportional to the electron density. The high occurrence "butterfly"-shaped band observed in Fig. 3 may be explained in this context as follows. The recombination rates in the F-region are lower than in the E-region, so the F-region electron density remains high throughout the late afternoonearly-evening, while the E-region density (and hence, conductance) drops off quickly with LT. Hence, this region of high density and low conductance corresponds to a region of high occurrence.

The high correlation between the electron density and echo occurrence observed in Fig. 6 also helps to explain why the nighttime bite-out of echo occurrence (at the top and the bottom) occurred at the same time as the bite-out in the electron density. As soon as F-region weakens after the sunset (with some considerable delay) so does the backscatter occurrence. This suggests that it is the electron density which controls the occurrence during the night time. The exact mechanism of this control is unclear, although the two potential candidates are under-refraction and the density-controlled GDI wave generation. A similar decrease in echo occurrence during winter midnight was observed by Milan et al. (1997). Those authors observed a decrease in both backscatter from the ground and the ionosphere. This would suggest that the decrease in occurrence is most likely due to under-refraction, as a decrease in irregularity generation should not affect the occurrence of ground scatter.

\subsection{Echo detection statistics: propagation effects and ir- regularity generation}

The final point of discussion relates to using the ionospheric echo occurrence parameter as a means to gain insights into both irregularity production and radio propagation processes. Generally speaking, the information about backscatter rates as a result of "convolution" of these two groups of processes is extremely valuable by itself, as it provides a tool to gauge when and where other valuable information (e.g. on convection velocity) can be obtained using a coherent scatter technique. At the same time, it has been noted very early on that backscatter rates are affected in a significant way by the ability of a HF radar ray to reach perpendicularity with the magnetic-field-aligned irregularities, so that the backscatter rates represent a lower limit on the occurrence of irregularities themselves (Ruohoniemi and Greenwald, 1997).

As discussed previously in Sect. 1, a common approach in studies employing radar echo occurrence statistics is to compute backscatter rates within a selected viewing area and analyse various occurrence trends, possibly complementing these data with the electron density information (e.g. Milan et al., 1997). Typically, the information about the slant range and hence about likely propagation mode $\left(\frac{1}{2}, 1 \frac{1}{2}\right.$ or 


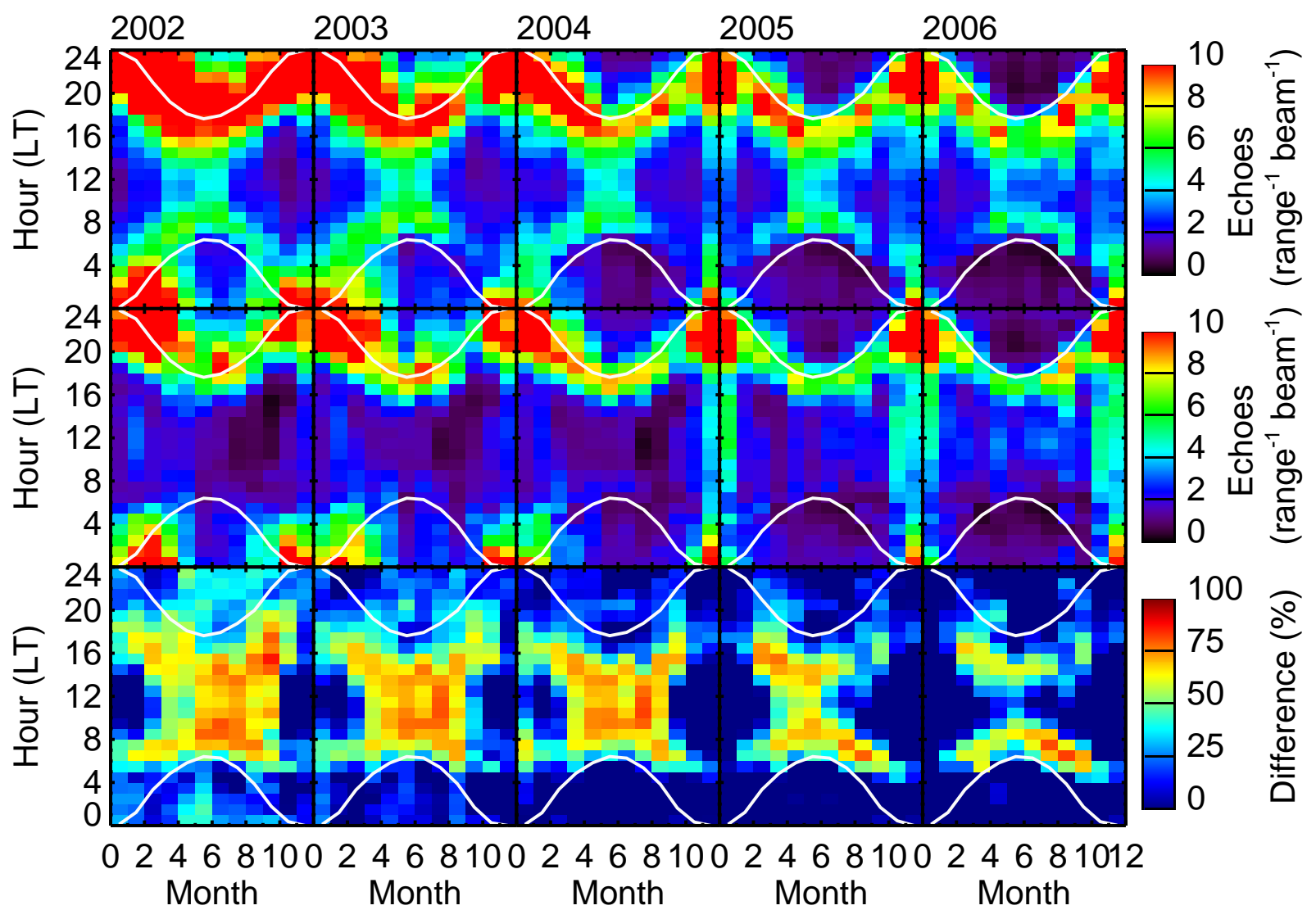

Fig. 7. Comparison of TIG echo occurrence statistics for ranges $765-3015 \mathrm{~km}$ (first row) and 765-1260 km (second row). The format of each panel is the same as in Fig. 3. The third row shows percentage difference between the two sets of measurements.

even $2 \frac{1}{2}$-hop) is considered explicitly as backscatter rates are computed for each range or MLAT gate (Ruohoniemi and Greenwald, 1997; Milan et al., 1997; Parkinson et al., 2003; Koustov et al., 2004). An alternative approach is to collect all range/MLAT bins together within a latitudinally-wide area and analyse the results with respect to their dependence on global drivers (Ballatore et al., 2000; Kane and Makarevich, 2010). In the current study, we have adopted the second approach, since we were interested in at least three kinds of effects (solar cycle, seasonal and diurnal), with the explicit consideration of the fourth dependence on range being challenging in this context. At the same time, we have also analysed the behaviour of the typical slant range in the same context (Fig. 3, second row) as discussed below.

The backscatter rates are always affected by the propagation conditions to some extent. One important propagation effect is expected when large amounts of ground- and seascatter are detected by the radar. The ground scatter is typically stronger than its ionospheric counterpart and, if present, it would effectively mask the ionospheric scatter even if the latter is present at the same range, thus reducing the backscatter rates. The extent of this effect would be largely determined by when and where the ground-scatter is observed, i.e. it will be more significant during the day and at farther ranges (e.g. Milan et al., 1997, and their Fig. 7). Conversely, at the ranges corresponding to the $\frac{1}{2}$-hop F-region mode (e.g. below $\sim 1260 \mathrm{~km}$ ) this effect is expected to be minimal.

Figure 3 indeed showed that the typical backscatter range did increase between sunrise and sunset (white curves), so that the daytime echo occurrence rates were likely to be affected. In attempt to further evaluate the extent of these effects, Fig. 7 presents the same analysis as in Fig. 3, but with an additional limit on range of $1260 \mathrm{~km}$ imposed in the second row. The third row panels show the percentage difference between the two sets of measurements. Visually, the enhanced occurrence near sunset in the first row was much less affected than the one near sunrise which virtually disappeared after sunrise in summer when ranges were restricted. From the third row of Fig. 7, the most significant differences were observed during the day, as expected, with the typical values of $50 \%-70 \%$. The differences were decreasing with the solar cycle. Overall, with the exception of the postsunrise summer echoes, which appear to be detected through the $1 \frac{1}{2}$-hop mode, all other features were present with the new range restrictions including the low occurrence during the day time and summer night time. 
We have also repeated the two analyses presented in Figs. 5 and 6, respectively, with new range restrictions, and results were largely similar to those in Sect. 3.4 (not presented here). One can conclude that the radar appeared to sample two different areas of the ionosphere during the day and night time. This was mostly likely due to ground scatter occupying the closest F-region ranges during the day time, when ionospheric density was enhanced, with ionospheric scatter being observed at farther ranges. At the same time, a subset of the daytime ionospheric echoes at the closest Fregion ranges was well representative of all daytime F-region echoes, suggesting that the irregularity generation processes were very similar at all considered ranges, at least as far as the features found in our analysis are concerned.

\section{Summary and conclusions}

Analysis of the solar cycle, seasonal and diurnal trends in the TIGER Bruny Island radar echo occurrence and model electron density and conductance under geomagnetically quiet conditions showed that:

1. The IRI-2007 model electron densities agree well with ionosonde measurements in the vicinity of the radar's footprint during quiet periods. The correlations between the model and measured $N m \mathrm{~F} 2$ values were particularly high at auroral and subauroral latitudes. Hence, the IRI model provides a reliable way of determining electron density during quiet periods except at very high latitudes.

2. The solar cycle effects in the F-region backscatter occurrence are strong and nonlinear. Systematic changes in the occurrence pattern occur from year to year that cannot be described by a single multiplicative factor relative to a solar minimum year. In particular, the solar cycle effects in the daytime occurrence are much weaker as compared to those during the night.

3. The seasonal and diurnal effects are strongly coupled. The diurnal variation of echo occurrence exhibits one or two peaks depending on the season and positions of the peaks change gradually from month to month. The occurrence is strongly peaked near midnight in summer, with the main peak moving towards dusk and secondary peak appearing near dawn in winter. The secondary peak gradually emerges from the post-midnight portion of the diurnal variation near the equinoxes.

4. During the day, the occurrence of F-region backscatter is controlled by both electrical conductance of the underlying ionosphere and the F-region electron density. The Pedersen conductance effects can be described as monotonic lowering of the upper and lower limits of the observed occurrence rates with conductance increase.
The upper (lower) limit is reached for large (low) Fregion densities for a given conductance. The conductance effects can be due to the perturbation electric fields being shorted by the highly conductive E-region, while the electron density variation can affect the echo detection rates by altering propagation conditions.

5. During the night, the occurrence of F-region backscatter is mostly controlled by the F-region electron density. In the late afternoon and evening sectors, the E-region and conductance quickly disappear with local time, while the F-region electron density and associated propagation effects would persist for some time. Due to relatively slow variations, the F-region electron density can enter into the range associated with favourable propagation conditions and/or can remain there for several hours after the sunset. This results in a band of high occurrence in the afternoon sector extending into the morning sector. The temporal extent and position of this band is dependent upon the season and solar cycle phase.

Acknowledgements. Operation of the TIGER Bruny Island radar was supported by a consortium of institutions: La Trobe University, University of Newcastle, Monash University, Australian Antarctic Division, ISR Division DSTO and IPS Radio and Space Services. The ionosonde data were supplied by the IPS Radio and Space Services, Australia. The authors thank A. J. Ribeiro for useful discussions regarding ionospheric scatter identification.

Topical Editor K. Kauristie thanks D. Danskin and R. Greenwald for their help in evaluating this paper.

\section{References}

Balan, N., Bailey, G. J., and Jayachandran, B.: Ionospheric evidence for a nonlinear relationship between the solar e.u.v. and $10.7 \mathrm{~cm}$ fluxes during an intense solar cycle, Planet. Space Sci., 41, 141-145, 1993.

Ballatore, P., Villain, J. P., Vilmer, N., and Pick, M.: The influence of the interplanetary medium on SuperDARN radar scattering occurrence, Ann. Geophys., 18, 1576-1583, doi:10.1007/s00585001-1576-2, 2000.

Benkevitch, L. V., Lyatsky, W. B., Koustov, A. V., Sofko, G. J., and Hamza, A. M.: Substorm onset times as derived from geomagnetic indices, Geophys. Res. Lett., 29, 1496, doi:10.1029/2001GL014386, 2002.

Bilitza, D. and Reinisch, B. W.: International Reference Ionosphere 2007: Improvements and new parameters, Adv. Space Res., 42, 599-609, 2008.

Carter, B. A. and Makarevich, R. A.: E-region decameter-scale plasma waves observed by the dual TIGER HF radars, Ann. Geophys., 27, 261-278, doi:10.5194/angeo-27-261-2009, 2009.

Chisham, G., Lester, M., Milan, S. E., Freeman, M. P., Bristow, W. A., Grocott, A., McWilliams, K. A., Ruohoniemi, J. M., Yeoman, T., Dyson, P. L., Greenwald, R. A., Kikuchi, T., Pinnock, M., Rash, J. P. S., Sato, N., Sofko, G. J., Villain, J.-P., and Walker, A. D. M.: A decade of the Super Dual Auroral Radar Network (SuperDARN): scientific achievements, new techniques and future directions, Surv. Geophys., 28, 33-109, 2007. 
Danskin, D. W., Koustov, A. V., Ogawa, T., Nishitani, N., Nozawa, S., Milan, S. E., Lester, M., and Andre, D.: On the factors controlling occurrence of F-region coherent echoes, Ann. Geophys., 20, 1385-1397, doi:10.5194/angeo-20-1385-2002, 2002.

Dyson, P. L. and Devlin, J. C.: The Tasman International Geospace Environment Radar, The Physicist (The Australian Institute of Physics), 37, 48-53, 2000.

Farley, D. T.: Incoherent Scatter Radar Probing, in: Modern Ionospheric Science, edited by: Kohl, H., Ruster, R., and Schlegel, K., pp. 415-439, Eur. Geophys. Soc., Katlenburg-Lindau, Germany., 1996.

Gonzalez, W. D., Joselyn, J. A., Kamide, Y., Kroehl, H. W., Rostoker, G., Tsurutani, B. T., and Vasyliunas, V. M.: What is a geomagnetic storm?, J. Geophys. Res., 99, 5771-5792, 1994.

Greenwald, R. A., Baker, K. B., Dudeney, J. R., Pinnock, M., Jones, T. B., Thomas, E. C., Villain, J.-P., Cerisier, J.-C., Senior, C., Hanuise, C., Hunsuker, R. D., Sofko, G., Koehler, J., Nielsen, E., Pellinen, R., Walker, A. D. M., Sato, N., and Yamagishi, H.: DARN/SuperDARN: A global view of the dynamics of highlatitude convection, Space Sci. Rev., 71, 763-796, 1995.

Greenwald, R. A., Oksavik, K., Erickson, P. J., Lind, F. D., Ruohoniemi, J. M., Baker, J. B. H., and Gjerloev, J. W.: Identification of the temperature gradient instability as the source of decameterscale ionospheric irregularities on plasmapause field lines, Geophys. Res. Lett., 331, L18105, doi:10.1029/2006GL026581, 2006.

Hanuise, C., Villain, J.-P., Cerisier, J. C., Senior, C., Ruohoniemi, J. M., Greenwald, R. A., and Baker, K. B.: Statistical study of high-latitude E region Doppler spectra obtained with SHERPA HF radar, Ann. Geophys., 9, 273-285, 1991.

Jayachandran, P. T., St.-Maurice, J.-P., MacDougall, J. W., and Moorcroft, D. R.: HF detection of slow long-lived E region plasma structures, J. Geophys. Res., 105, 2425-2442, 2000.

Kane, T. A. and Makarevich, R. A.: HF radar observations of the F region ionospheric plasma response to Storm Sudden Commencements, J. Geophys. Res., 115, A07320, doi:10.1029/2009JA014974, 2010.

Kelley, M. C.: The Earth's Ionosphere, Academic Press, San Diego, Second edn., 2009.

Kelley, M. C., Vickrey, J. F., Carlson, C. W., and Torbert, R.: On the origin and spatial extent of high-latitude $\mathrm{F}$ region irregularities, J. Geophys. Res., 87, 4469-4475, 1982.

Keskinen, M. J. and Ossakow, S. L.: Theories of high-latitude ionospheric irregularities: A review, Radio Sci., 18, 1077-1091, 1983.

Koustov, A. V., Sofko, G. J., André, D., Danskin, D. W., and Benkevitch, L. V.: Seasonal variation of HF radar F region echo occurrence in the midnight sector, J. Geophys. Res., 109, A06305, doi:10.1029/2003JA010337, 2004.
Kumar, V. V., Makarevich, R. A., Kane, T. A., Ye, H., Devlin, J. C., and Dyson, P. L.: On the spatiotemporal evolution of the ionospheric backscatter during magnetically disturbed periods as observed by the TIGER Bruny Island HF radar, J. Atmos. Sol. Terr. Phys., 73, 1940-1952, 2011.

Makarevich, R. A.: On the occurrence of high-velocity E-region echoes in SuperDARN observations, J. Geophys. Res., 115, A07302, doi:10.1029/2009JA014698, 2010.

Makarevich, R. A., Kellerman, A. C., Devlin, J. C., Ye, H., Lyons, L. R., and Nishimura, Y.: SAPS intensification during substorm recovery: A multi-instrument case study, J. Geophys. Res., 116, A11311, doi:10.1029/2011JA016916, 2011.

Milan, S. E. and Lester, M.: Spectral and flow angle characteristics of backscatter from decametre irregularities in the auroral electrojets, Adv. Space Res., 23, 1773-1776, 1999.

Milan, S. E., Yeoman, T. K., Lester, M., Thomas, E. C., and Jones, T. B.: Initial backscatter occurrence statistics from the CUTLASS HF radars, Ann. Geophys., 15, 703-718, doi:10.1007/s00585-997-0703-0, 1997.

Parkinson, M. L., Devlin, J. C., Ye, H., Waters, C. L., Dyson, P. L., Breed, A. M., and Morris, R. J.: On the occurrence and motion of decametre-scale irregularities in the sub-auroral, auroral, and polar cap ionosphere, Ann. Geophys., 21, 1847-1868, doi:10.5194/angeo-21-1847-2003, 2003.

Ponomarenko, P. V., Waters, C. L., and Menk, F. W.: Effects of mixed scatter on SuperDARN convection maps, Ann. Geophys. 26, 1517-1523, doi:10.5194/angeo-26-1517-2008, 2008.

Ribeiro, A. J., Ruohoniemi, J. M., Baker, J. B. H., Clausen, L. B. N., de Larquier, S., and Greenwald, R. A.: A new approach for identifying ionospheric backscatter in midlatitude SuperDARN HF radar observations, Radio Sci., 46, RS4011, doi:10.1029/2011RS004676, 2011.

Robinson, R. M. and Vondrak, R. R.: Measurements of E region ionization and conductivity produced by solar illumination at high latitudes, J. Geophys. Res., 89, 3951-3956, 1984.

Ruohoniemi, J. M. and Greenwald, R. A.: Rates of scattering occurrence in routine $\mathrm{HF}$ radar observations during solar cycle maximum, Radio Sci., 32, 1051-1070, 1997.

Tsunoda, R. T.: High-latitude F region irregularities - A review and synthesis, Rev. Geophys., 26, 719-760, 1988.

Vickrey, J. F. and Kelley, M. C.: The effects of a conducting E layer on classical $\mathrm{F}$ region cross-field plasma diffusion, J. Geophys. Res., 87, 4461-4468, 1982.

Wild, J. A. and Grocott, A.: The influence of magnetospheric substorms on SuperDARN radar backscatter, J. Geophys. Res., 113, A04308, doi:10.1029/2007JA012910, 2008. 